\title{
Desenvolvimento de Tradução para Português do Patient Enablement Instrument
}

\author{
Portuguese Translation of the Patient Enablement Instrument \\ Inês Pintalhão*, Philippe Botas**, Carolina Pereira**, Luiz Miguel Santiago***
}

Pontos-chave:

A capacitação é o ganho que o doente adquire numa consulta para poder compreender e lidar com a sua doença. Pode ser atualmente medido por meio de questionário.

Realizou-se trabalho que venha a permitir adaptar a escala para Portugal.

\section{Resumo}

Introdução: A capacitação (enablement) traduz-se pelo ganho que o doente adquire numa consulta para poder compreender e lidar com a sua doença. O Patient Enablement Instrument (PEI) é um instrumento que permite analisar e medir o resultado de uma consulta em função da capacitação do consulente.

Objetivos: Desenvolver uma tradução portuguesa do PEl.

Métodos: Foi efetuada uma tradução do questionário PEI para português, designada por Instrumento de Capacitação do Consulente (ICC), segundo as regras internacionalmente aceites para este género de tarefas. O ICC foi aplicado a 55 consulentes, para validade de conteúdo, pela linguística e conhecimento de problemas de percepção.

Resultados: A tradução do questionário mostrou conformidade linguística e semântica com o instrumento original e sem problemas na sua interpretação populacional.

Discussão e Conclusão: Foi possível desenvolver uma tradução portuguesa do PEl. Deve agora ser desenvolvida a validação para a população portuguesa.

Palavras-Chave: Medicina Geral e Familiar, Cuidados de Saúde Primários, capacitação, Patient Enablement Instrument, consulta.

\section{Abstract}

Introduction: Enablement means the gain a patient acquires in a medical consultation to understand and cope with his diease or medical condition. The Patient Enablement Instrument (PEI) is an instrument that allows the measurement and analysis of the result of a medical consultation.

Objetiv: To develop a portuguese translation oh the Patient Enablement Instrument.

Methods: A English to Portuguese translation of the PEI was made according to the best international scientific proceedings. A back to english translation was made and the translated PEI was then applied to 55 portuguese patients to know about linguistic perception problems.

Results: The portuguese translation of the PEI has shown good match with the english one and free of important perception problems.

Conclusion: A portuguese translation of the PEI was made. Its validation process must now de performed.

Key-words: General Practice Medicine, Primary Heath Care, Enablement, Patient Enablement Instrument, consultation.

\footnotetext{
*Aluna de Mestrado Integrado da Faculdade de Medicina da Universidade de Coimbra

${ }^{* *}$ MD, Interno de Especialidade de Medicina Geral e Familiar, USF Topázio, ACES Baixo Mondego I

${ }^{* * *}$ MD, PhD, Assistente Graduado Sénior de Medicina Geral e Familiar, USF Topázio, ACES Baixo Mondego I e Professor na Faculdade de Ciências da Saúde da Universidade da Beira Interior
} 


\section{Introdução}

Os Cuidados de Saúde Primários (CSP) constituem a principal estrutura de um sistema de saúde ${ }^{1}$, quer pela diversidade de problemas que abrangem, quer pela dimensão de problemas de saúde que resolvem. Na área de Medicina Geral e Familiar (MGF) o médico recebe diariamente um número importante de consulentes aos quais, através de um complexo sistema de percepções, análises e interpretações, procede a uma avaliação cuidadosa do quadro e a medidas de intervenção ${ }^{2}$. Desta forma, uma consulta consiste não só na tomada de decisões partilhadas entre o médico e o consulente em relação ao seu problema ${ }^{3}$, mas também num espaço em que o consulente expõe as suas preocupações, esperando do médico compreensão e apoio, face às suas expectativas.

Howie et al introduziram pela primeira vez o termo "capacitação" (enablement), que se refere ao ganho que efetivamente o consulente adquire numa consulta para poder compreender e lidar com a sua doença o melhor possivel $^{4,5}$. Neste âmbito os mesmos autores criaram um instrumento que permite analisar e medir o resultado de uma consulta em função da capacitação do consulente - Patient Enablement Instrument (PEI) ${ }^{6}$. Este questionário foi já validado e aplicado em diversos países, como a Inglaterra, Croácia, Holanda, Polónia, Espanha e China ${ }^{7}$, que demonstraram que um aumento da capacitação se pode traduzir numa maior adesão à terapêutica e melhoria de resultados a longo prazo.

Em Portugal, não há resultados publicados sobre o conceito de capacitação assim como sobre a utilização de instrumentos para a sua avaliação nomeadamente do PEl.

\section{Objectivo}

Desenvolver uma tradução para português do Enablement Instrument (PEI) e a denominar de Instrumento de Capacitação do Consulente (ICC).

\section{Metodologia}

Adaptação e tradução do questionário PEI para língua portuguesa

Foi solicitada aos autores do PEl, por escrito, para a morada constante do artigo original, a necessária autorização para a realização do estudo. Apesar de não obtermos resposta ao fim de seis meses, como houve aprovação pela Comissão de Ética para a realização do trabalho, e dado a escala estar já publicada em vários contextos geográficos, julgou-se ser uma perda, para o resultado da saúde em Portugal, a não realização do presente trabalho.

A tradução do questionário para língua portuguesa envolveu um painel de colaboradores e foi efetuada segundo um método internacionalmente recomendado ${ }^{8}$, primeiro pela tradução da versão original inglesa para língua portuguesa por dois tradutores profissionais de língua nativa portuguesa com habilitações na área da saúde, independentemente um do outro. Uma equipa constituída por uma socióloga, uma doutoranda em Comunicação e Saúde, um médico e uma assistente social efetuou a revisão da tradução, criando assim a primeira versão do $\mathrm{PEI}$ e adaptando a melhor tradução portuguesa para o espírito do questionário em inglês.

O passo seguinte consistiu na tradução desta mesma versão novamente para língua inglesa, realizada por dois tradutores profissionais de língua nativa inglesa. Outra equipa constituída por médico, socióloga e psicóloga avaliou se a tradução para inglês estava conforme o conteúdo e a linguística da versão original.

Procedeu-se depois a uma avaliação da tradução portuguesa por uma perita em linguística, de modo a corrigir, inequivocamente, o fraseado português.

O PEI é constituído por 6 perguntas, nas quais é inquirido ao doente, após a consulta com o seu médico, os seguintes tópicos: "capacidade de lidar com a vida", "capacidade de compreender a sua doença”, "capacidade de lidar com a sua doença”, "capacidade de se manter saudável”, "confiança em relação à sua saúde" e "capacidade de se ajudar a si próprio". As respostas variam entre "muito melhor", "melhor" ou "igual ou pior" para as quatro primeiras afirmações, e entre "muito mais", "mais" ou "igual ou menos", para as duas últimas, respetivamente.

Realizou-se ainda a aplicação do PEI/ICC, num pré-teste, a 55 consulentes da USF, sendo o questionário preenchido pelos próprios entrevistados à saída da consulta, durante o mês de Abril de 2013, para verificar dificuldades de interpretação ou de linguagem portuguesa, bem como para registar o tempo de preenchimento.

\section{Análise das respostas do painel inicial}

O tratamento estatístico dos dados colhidos foi realizado com o auxílio do software "SPSS software for Windows - version 19.0" (SPSS Inc, Chicago, IL), sendo realizada estatística descritiva e análise inferencial, após verifi- 
cação da normalidade dos dados. Para análise de variáveis nominais foi utilizado o teste $x 2$. Foi utilizado o teste não para métrico Kruskal-Wallis para verificar a diferença entre os vários tempos de análise, definindo-se como estatisticamente significativo o valor de $p<0,05$.

\section{Resultados}

Após obtenção de autorização da Comissão de Ética da Administração Regional do Centro (ARS Centro) e para verificação da aceitabilidade e de problemas com a formulação das perguntas estudou-se uma amostra não probabilística de consulentes que saíam dos gabinetes médicos da USF, não sabendo os médicos que o estudo se estava a realizar. A investigadora abordou os consulentes devidamente identificada e solicitou o preenchimento por escrito após obtenção de assinatura do respectivo consentimento informado. Foram inquiridos 55 consulentes, sendo 17 indivíduos do género masculino (30,9\%) e 38 do género feminino (69,1\%), com idades compreendidas entre os 21 e os 88 anos (média de 54,29 anos, mediana de 52 e moda de 32). 69,1\% dos doentes entrevistados enquadravam-se no grupo etário com idade inferior a 65 anos. No que diz respeito à Toma Diária de Medicamentos (TDM), 69,1\% dos doentes da amostra referia-a. Relativamente ao grau de instrução, 5,5\% dos indivíduos apresentava grau de escolaridade baixo - inferior ao $9^{\circ}$ ano -, 52,7\% grau médio - equivalente ao $9^{\circ}$ ano - e 41,8\% grau elevado de escolaridade, superior ao $9^{\circ}$ ano, segundo o Quadro I.
Quadro I: Caracterização da amostra estudada para validação do questionário.

\begin{tabular}{|c|c|}
\hline Variável & $n(\%)$ \\
\hline \multicolumn{2}{|c|}{ Grupo Etário } \\
\hline$<65$ anos & $38(69,1)$ \\
\hline$\geq 65$ anos & $17(30,9)$ \\
\hline \multicolumn{2}{|c|}{ Género } \\
\hline Homem & $17(30,9)$ \\
\hline Mulher & $38(69,1)$ \\
\hline \multicolumn{2}{|c|}{ Grau de Instrução } \\
\hline Baixo & $3(5,5)$ \\
\hline Médio & $29(52,7)$ \\
\hline Alto & $23(41,8)$ \\
\hline \multicolumn{2}{|c|}{ Toma Diária de Medicamentos } \\
\hline Sim & $38(69,1)$ \\
\hline Não & $17(30,9)$ \\
\hline
\end{tabular}

As respostas dadas pelos entrevistados às afirmações do questionário, em função das variáveis consideradas, estão representadas no Quadro II.

A média de tempo de preenchimento do questionário foi de 3 a 5 minutos nos indivíduos de grau de escolaridade médio e alto, e de 5 a 10 minutos nos consulentes com grau de escolaridade mais baixo.

Numa análise inferencial, apenas se verificou haver diferença estatisticamente significativa para a questão "Capaz de se manter saudável", em que os doentes que referiram Toma Diária de Medicamentos (TDM) se sentiam "igual ou pior", após a consulta com o médico [18 $(85,7 \%)$ TDM vs $3(14,3 \%)$ não TDM, p=0,042]. 
Quadro II: Respostas às afirmações em função das variáveis consideradas.

\begin{tabular}{|c|c|c|c|c|c|c|c|c|c|}
\hline \multirow[b]{2}{*}{ Afirmação } & \multicolumn{2}{|c|}{ Grupo Etário } & \multicolumn{2}{|c|}{ Género } & \multicolumn{2}{|c|}{$\begin{array}{l}\text { Toma Diária de } \\
\text { Medicamentos }\end{array}$} & \multicolumn{3}{|c|}{ Grau de Instrução } \\
\hline & $\begin{array}{c}<5 \text { anos } \\
n(\%)\end{array}$ & $\begin{array}{c}\geq 65 \text { anos } \\
n(\%)\end{array}$ & $\begin{array}{c}\text { Homem } \\
\text { n (\%) }\end{array}$ & $\begin{array}{l}\text { Mulher } \\
\text { n (\%) }\end{array}$ & $\begin{array}{l}\text { Sim } \\
n(\%)\end{array}$ & $\begin{array}{l}\text { Não } \\
\text { n (\%) }\end{array}$ & $\begin{array}{l}\text { Baixo } \\
\text { n (\%) }\end{array}$ & $\begin{array}{l}\text { Médio } \\
\text { n (\%) }\end{array}$ & $\begin{array}{l}\text { Alto } \\
n(\%)\end{array}$ \\
\hline \multicolumn{10}{|c|}{ Capaz de lidar com a vida } \\
\hline Muito Melhor & $8(21,1)$ & $2(11,8)$ & $2(20,0)$ & $8(80,0)$ & $7(70,0)$ & $3(30,0)$ & $0(0,0)$ & $5(17,2)$ & $5(21,7)$ \\
\hline Melhor & $21(55,3)$ & $7(41,2)$ & $8(28,6)$ & $20(71,4)$ & $16(57,1)$ & $12(42,9)$ & $1(33,3)$ & $11(37,9)$ & $16(69,6)$ \\
\hline Igual ou pior & $9(23,7)$ & $8(47,1)$ & $7(41,2)$ & $10(58,8)$ & $15(88,2)$ & $2(11,8)$ & $2(66,7)$ & $13(44,8)$ & $2(8,7)$ \\
\hline \multicolumn{10}{|c|}{ Capaz de compreender a sua doença } \\
\hline Muito Melhor & $11(28,9)$ & $2(11,8)$ & $5(35,7)$ & $9(64,3)$ & $9(64,3)$ & $5(35,7)$ & $0(0,0)$ & $5(17,2)$ & $9(39,1)$ \\
\hline Melhor & $21(55,3)$ & $5(29,4)$ & $8(30,8)$ & $18(69,2)$ & $15(57,7)$ & $11(42,3)$ & $0(0,0)$ & $13(44,8)$ & $13(56,5)$ \\
\hline Igual ou pior & $7(18,4)$ & $8(47,1)$ & $4(26,7)$ & $11(73,3)$ & $14(93,3)$ & $1(6,7)$ & $3(100,0)$ & $11(37,9)$ & $1(4,3)$ \\
\hline \multicolumn{10}{|c|}{ Capaz de lidar com a sua doença } \\
\hline Muito Melhor & $11(28,9)$ & $2(11,8)$ & $3(23,1)$ & $10(76,9)$ & $9(69,2)$ & $4(30,8)$ & $0(0,0)$ & $5(17,2)$ & $8(34,8)$ \\
\hline Melhor & $22(57,9)$ & $10(58,8)$ & $9(28,1)$ & $23(71,9)$ & $20(62,5)$ & $12(37,5)$ & $2(66,7)$ & $16(55,2)$ & $14(60,9)$ \\
\hline Igual ou pior & $5(13,2)$ & $5(29,4)$ & $5(50,0)$ & $5(50,0)$ & $9(90,0)$ & $1(10,0)$ & $1(33,3)$ & $8(27,6)$ & $1(4,3)$ \\
\hline \multicolumn{10}{|c|}{ Capaz de se manter saudável } \\
\hline Muito Melhor & $10(26,3)$ & $1(5,9)$ & $1(9,1)$ & $10(90,9)$ & $6(54,5)^{a}$ & $5(45,5)^{\mathrm{a}}$ & $0(0,0)$ & $3(10,3)$ & $8(34,8)$ \\
\hline Melhor & $17(44,7)$ & $6(35,3)$ & $10(43,5)$ & $13(56,5)$ & $14(60,9)^{a}$ & $9(39,1)^{a}$ & $0(0,0)$ & $13(44,8)$ & $10(43,5)$ \\
\hline Igual ou pior & $11(28,9)$ & $10(58,8)$ & $6(28,6)$ & $15(71,4)$ & $18(85,7)^{\mathrm{a}}$ & $3(14,3)^{a}$ & $3(100,0)$ & $13(44,8)$ & $5(21,7)$ \\
\hline \multicolumn{10}{|c|}{ Confiante em relação à sua saúde } \\
\hline Muito Melhor & $11(28,9)$ & $2(11,8)$ & $3(23,1)$ & $10(76,9)$ & $7(53,8)$ & $6(46,2)$ & $1(33,3)$ & $7(24,1)$ & $5(21,7)$ \\
\hline Melhor & $18(47,4)$ & $8(47,1)$ & $10(38,5)$ & $16(61,5)$ & $18(69,2)$ & $8(30,8)$ & $0(0,0)$ & $13(44,8)$ & $13(56,5)$ \\
\hline Igual ou pior & $9(23,7)$ & $7(41,2)$ & $4(25,0)$ & $12(75,0)$ & $13(81,3)$ & $3(18,8)$ & $2(66,7)$ & $9(31,0)$ & $5(21,7)$ \\
\hline \multicolumn{10}{|c|}{ Capaz de se ajudar a si próprio } \\
\hline Muito Melhor & $11(28,9)$ & $4(23,5)$ & $3(20,0)$ & $12(80,0)$ & $9(23,7)$ & $6(35,3)$ & $0(0,0)$ & $9(31,0)$ & $6(21,6)$ \\
\hline Melhor & $17(44,7)$ & $6(35,3)$ & $8(34,8)$ & $15(65,2)$ & $14(36,8)$ & $9(52,9)$ & $1(33,3)$ & $10(34,5)$ & $12(52,2)$ \\
\hline Igual ou pior & $10(26,3)$ & $7(41,2)$ & $6(35,3)$ & $11(64,7)$ & $15(39,5)$ & $12(11,8)$ & $3(100,0)$ & $10(34,5)$ & $5(21,7)$ \\
\hline
\end{tabular}

\section{Discussão}

O presente trabalho teve como objetivo o desenvolvimento de uma tradução portuguesa do questionário PEI dado que acerca deste instrumento não se conhecem publicações em Portugal.

A introdução e abordagem ao "recente" conceito de capacitação, passível de ser medida por este instrumento, tem vindo a revelar-se extremamente útil na avaliação de resultados das consultas noutros países, de modo a criar táticas, da parte do médico e das estruturas de
CSP, que visem uma melhoria desses mesmos resultados. De facto, tem sido provado que os estudos em que o consulente se coloca no papel de observador, relatando as suas experiências e perspectivas, constituem hoje a melhor prática na avaliação dos cuidados de saúde prestados $^{9}$. Este trabalho a ser continuado, poderá revelar se, para o contexto da prática da MGF em Portugal, as consequências do conhecimento da capacitação são importantes e semelhantes às já conhecidas.

A tradução do questionário e os posteriores procedimentos permitiram cumprir os passos necessários à 
validação da tradução linguística deste instrumento. As principais dúvidas no preenchimento do questionário consistiram na dificuldade, por parte dos consulentes, em perceber que as respostas deveriam ser dadas em função da consulta da qual tinham acabado de sair, e não relativamente às suas preocupações e sentimentos prévios à consulta. A principal crítica apontada ao ICC consistiu no facto de os consulentes considerarem as questões genéricas e pouco objetivas, levando-os a ter alguma dificuldade de interpretação. No entanto, este problema não foi julgado impeditivo de preenchimento de resposta pelos consulentes.

Deverá agora ser desenvolvido o processo de validação populacional para patologias específicas através da metodologia mais indicada como a aplicação e reaplicação em tempo posterior ou por diferente forma de aplicação para se saber de não diferença de resultado em função da forma de aplicação.

\section{Referências Bibliográficas}

1. Poulton, B.C., Use of the consultation satisfaction questionnaire to examine patients' satisfaction with general practitioners and community nurses: reliability, replicability and discriminant validity. Br J Gen Pract, 1996. 46(402): p. 26-31.

2. Santiago, L.M., C. Neves, and L. Constantino, [Patient's relationship with the medical prescription - an observational study in urban populations in the ambulatory setting of General Practice/Family Medicine in the centre of Portugal]. Acta Med Port, 2010. 23(5): p. 755-60.

3. Mercer, S.W., et al., Patient enablement requires physician empathy: a cross-sectional study of general practice consultations in areas of high and low socioeconomic deprivation in Scotland. BMC Fam Pract, 2012. 13: p. 6.

4. Howie, J.G., D.J. Heaney, and M. Maxwell, Measuring quality in general practice. Pilot study of a needs, process and

\section{Conclusões}

O presente estudo permitiu desenvolver a tradução para portuguès do questionário PEI que apelidámos de Instrumento de Capacitação do Consulente (ICC).

O PEI/ICC deve agora ser validado em processo específico.

\section{Agradecimentos}

Os autores desejam agradecer a todos quantos participaram nos painéis de peritos e aos tradutores, mesmo que todos tenham solicitado anonimato.

E desejam igualmente agradecer aos consulentes que desinteressadamente deram algum do seu tempo a este processo.

outcome measure. Occas Pap R Coll Gen Pract, 1997(75): p. i-xii, 1-32.

5. Howie, J.G., et al., Quality at general practice consultations: cross sectional survey. Bmj, 1999. 319(7212): p. 738-43.

6. McKinstry, B., I. Colthart, and J. Walker, Can doctors predict patients' satisfaction and enablement? A cross-sectional observational study. Fam Pract, 2006. 23(2): p. 240-5.

7. Lam, C.L., et al., A pilot study on the validity and reliability of the Patient Enablement Instrument (PEI) in a Chinese population. Fam Pract, 2010. 27(4): p. 395-403.

8. Wild, D., et al., Principles of Good Practice for the Translation and Cultural Adaptation Process for Patient-Reported Outcomes (PRO) Measures: report of the ISPOR Task Force for Translation and Cultural Adaptation. Value Health, 2005. 8(2): p. 94-104.

9. Ferreira, P.L., Avaliação dos doentes de cuidados primários. Revista Portuguesa de Clínica Geral, 2001. 17: p. 15-45.

\section{Conflito de Interesses}

Os autores declaram não ter conflito de interesses em relação a este artigo.

\section{Endereço para Correspondência:}

Philippe Botas

USF Topázio, Rua Dr João Pinheiro s/n

3020-069 Coimbra

Email: phbotas@gmail.com 